Research Article

\title{
An Integrated Approach for Cancer Survival Prediction Using Data Mining Techniques
}

\author{
Ishleen Kaur $\mathbb{D}^{1},{ }^{1}$ M. N. Doja, ${ }^{1}$ Tanvir Ahmad $\left(\mathbb{D},{ }^{1}\right.$ Musheer Ahmad $\mathbb{D}^{1},{ }^{1}$ Amir Hussain $\mathbb{D}^{2}{ }^{2}$ \\ Ahmed Nadeem $\mathbb{D}^{3},{ }^{3}$ and Ahmed A. Abd El-Latif $\mathbb{D}^{4}$ \\ ${ }^{1}$ Department of Computer Engineering, Jamia Millia Islamia, New Delhi 110025, India \\ ${ }^{2}$ School of Computing, Edinburgh Napier University, Merchiston Campus, Edinburgh, Scotland EH10 5DT, UK \\ ${ }^{3}$ Department of Pharmacology \& Toxicology, College of Pharmacy, King Saud University, \\ PO Box 2455, Riyadh 11451, Saudi Arabia \\ ${ }^{4}$ Department of Mathematics and Computer Science, Faculty of Science, Menoufia University, Shibin El Kom 32511, Egypt
}

Correspondence should be addressed to Ahmed A. Abd El-Latif; a.rahiem@gmail.com

Received 4 November 2021; Accepted 27 November 2021; Published 28 December 2021

Academic Editor: Anastasios D. Doulamis

Copyright $\odot 2021$ Ishleen Kaur et al. This is an open access article distributed under the Creative Commons Attribution License, which permits unrestricted use, distribution, and reproduction in any medium, provided the original work is properly cited.

\begin{abstract}
Ovarian cancer is the third most common gynecologic cancers worldwide. Advanced ovarian cancer patients bear a significant mortality rate. Survival estimation is essential for clinicians and patients to understand better and tolerate future outcomes. The present study intends to investigate different survival predictors available for cancer prognosis using data mining techniques. Dataset of 140 advanced ovarian cancer patients containing data from different data profiles (clinical, treatment, and overall life quality) has been collected and used to foresee cancer patients' survival. Attributes from each data profile have been processed accordingly. Clinical data has been prepared corresponding to missing values and outliers. Treatment data including varying time periods were created using sequence mining techniques to identify the treatments given to the patients. And lastly, different comorbidities were combined into a single factor by computing Charlson Comorbidity Index for each patient. After appropriate preprocessing, the integrated dataset is classified using appropriate machine learning algorithms. The proposed integrated model approach gave the highest accuracy of $76.4 \%$ using ensemble technique with sequential pattern mining including time intervals of 2 months between treatments. Thus, the treatment sequences and, most importantly, life quality attributes significantly contribute to the survival prediction of cancer patients.
\end{abstract}

\section{Introduction}

Cancer, along with coronary heart diseases, accounts for most deaths globally (the top 10 causes of death [1]). The incidence rate of cancer has increased over the past few decades. It has been estimated that 1 in 9 Indians will develop cancer during their lifetime. According to GLOBOCAN, India recorded the highest number of deaths globally in ovarian cancer. Ovarian cancer is the third most common site of cancer among women in India. It is also the third most commonly occurring gynecologic cancer worldwide and has the worst mortality rate. Clinicians and scientists have been conducting great experiments and research to predict cancer patients' survivability $[2,3]$. Yet, there are no quality survival estimation predictors available. Survival estimation predictors are essential for clinicians to precisely adopt the treatments and medications for the patients.

Data-driven prediction techniques can assist in a better cancer prognosis model. Since its origin, data mining techniques have been efficaciously used in many healthcare research kinds, especially cancer management $[4,5]$. The medical models based on data mining techniques can capture intricate details and patterns in data. Several studies involve online datasets like UCI machine learning, SEER [6], and TCGA [7]. However, these datasets only cover datasets from western countries or only from a limited area. Although the number of instances in online datasets is large, these might not capture the region-specific analyses. It has 
been proven in past studies that race and region can play a significant role in the survivability of cancer patients [8]. Conversely, clinical studies having fewer instances can capture more local aspects of cancer patients and their management. The present research focuses on various attributes that can be significant predictors in estimating the survival of advanced ovarian carcinoma patients that are mostly unavailable in online datasets.

The existing literature focuses on including different clinical attributes like age, CA-125 levels, histology, and stage to investigate the survivability and mortality of ovarian cancer patients [9]. Some of the researchers also intended to explore the outcome for patients treated with neoadjuvant chemotherapy (NACT) [10] or surgery [11]. Nonetheless, the current literature lacks proper research that may give insights into ovarian cancer survival using machine learning approaches and since its initiation, machine learning technology has progressed a lot and is proven to provide good results in almost every area. Some studies performed statistical analysis to find the correlation of treatments with survival [10]. But most of these studies are a part of clinical trials having a controlled environment. A retrospective study in an uncontrolled setting with a variety of participants can point out some useful insights that might not be possible with a clinical trial dataset. Also, to the best of the authors' knowledge, no existing literature emphasized the different sequences of treatments for ovarian cancer patients. Furthermore, various comorbidities can play an essential role in the overall health of patients [12]. The present study involves recording and using some relevant predictors for survival analysis of cancer patients and clinical attributes. These attributes were not available in any online datasets. The collected and processed features can be used and extended to survive any cancer or other serious condition study.

The present study aims to identify the significance of different predictors for advanced ovarian carcinoma patients. An integrated model using attributes from different data profiles can assist in a robust model for predicting survival outcome of patients. The attributes from different data profiles have been collected from a cancer hospital and processed accordingly. Cancer patients are given multiple lines of treatment to prolong their survival. The present study is an attempt to identify the different lines of treatments given to ovarian cancer patients using sequence mining approaches. These treatments and the estimated time elapsed among treatments might contribute some valuable perceptions to the survival of patients. Previous literature has also acknowledged the association of time between treatments with prognosis in patients with ovarian carcinoma [13]. Life quality attributes like performance status and comorbidities also have a significant impact on any person's survival. These attributes have been explored in the study to examine their effect on survival.

The remainder of the study is structured as follows: Section 2 presents a brief background on ovarian cancer and its prevalence in India. Section 3 provides some of the existing literature on ovarian cancer survival analysis. Section 4 explains in detail the dataset and the proposed methodology in the study. The study's results and discussion and its comparison to the existing methods are given in Section 5. Section 6 discusses some of the study's limitations, and the conclusion is presented in Section 7.

\section{Background}

2.1. Ovarian Cancer. Ovarian cancer has the worst mortality in all gynecologic cancers. Overweight and obese women have a higher risk of ovarian cancer [14]. Age is also a significant factor in cancer incidence. While its incidence rates have remained constant in some European countries, Asia has experienced increased incidence rates from the past few decades [15]. Survival rates are less than $20 \%$ in Indian women. According to a report, $50 \%$ of India's total ovarian cancer cases occurred at 45-65 years [16]. Though, most of the western countries have a median range of more than 60 [17].

Ovarian cancer can have around 90\% survival rates if detected in the early stage. However, reports have shown that most patients are diagnosed in later stages, with survival less than $40 \%$ (SEER Program). This is why it is also known as "silent killer" because more than $60 \%$ of the cases are diagnosed at advanced stages (Stages III and IV). Epithelial ovarian cancer is the most common, including high-grade serous, low-grade serous, endometrioid, clear cell, and mucinous. Patients diagnosed in most advanced ovarian cancer cases are provided with multiple lines of treatment. These include cytoreductive surgery (CRS) with adjuvant chemotherapy, or neoadjuvant chemotherapy (NACT) with Interval debulking surgery (IDS) and adjuvant chemotherapy, or hormonal therapy or chemotherapy [18].

2.2. Sequence Mining. A sequence 'seq' is a collection of ordered symbols. |seq| denotes the length of the sequence [19]. A substring of a sequence is a collection of consecutive symbols of the sequence. However, in a subsequence, the symbols need not be consecutive. For example, if PQRS is a sequence with symbols $\{\mathrm{P}, \mathrm{Q}, \mathrm{R}, \mathrm{S}\}$, then both $\mathrm{PQS}$ and $\mathrm{PQR}$ can be subsequences of the sequence. But PQS is not a substring of the mentioned sequence. Sequence mining refers to identifying frequently occurring subsequences from a database of sequences. The user determines the term "frequent" by varying the support of the sequences. Support of 0.5 suggests that the database should contain a subsequence in at least $50 \%$ of the sequences.

Researchers have devised several sequence mining algorithms. Generalized Sequential Pattern (GSP) [20] is one of the first sequence mining algorithms formulated on the basis of Apriori algorithm [21]. GSP works by identifying the subsequences by scanning the dataset and computing their support. Subsequences with support less than the threshold support are removed from further analysis. For $k$ length sequences, GSP scans the dataset $k$ times. Once the frequent sequence at level $k$ ( $k$ length sequence) is found, a candidate for length $k+1$ is generated. Various other researchers also tried to formulate sequence mining algorithms with less time and space complexity. SPADE and PrefixSpan are examples of such algorithms [22, 23]. 


\section{Related Work}

Several researchers tried to analyze advanced ovarian cancer patients' survival using statistical and conventional survival methods concerning different survival estimators. Vincent et al. [10] used univariate analysis to identify the prognostic factors for stage $3 \mathrm{c}$ or $4 \mathrm{a}$ ovarian cancer patients. The dataset was collected from 11 French centers and included 483 patients who were treated with NACT followed by surgery. Univariate analysis showed that the absence of cytoreductive surgery (CRS) was associated with worse survival. Similarly, CA-125 value higher or equal to $3000 \mathrm{U} / \mathrm{ml}$ had decreased overall survival.

Deng et al. [24] used data from the online dataset SEER (SEER Program) to analyze the survival based on metastatic site for stage 4 patients. Due to the publicly available online dataset, the number of patients is higher than that in other clinical studies. After various inclusions and exclusions, the data analyzed consisted of 1481 patients. Univariate and multivariate analyses showed that the most common sites of metastasis are liver followed by lymph nodes. For patients with lung metastases only, patients who received chemotherapy had a higher survival than those who did not receive chemotherapy. Surgery was also associated with higher survival rates in patients with lymph nodes and liver metastases, but it was not a significant self-determining aspect in patients having lung metastasis. Akhavan et al. [12] also conducted statistical chi squared and Student's test on a dataset collected from Tehran to investigate the effect of diabetes on ovarian cancer survival. The histology considered in particular was epithelial carcinoma. The results suggested that the patients having diabetes had poor overall and progression free survival than those without diabetes.

In a more recent study [25], the authors collected a dataset of around 460 patients from a cancer center to compare white women's survival with black women. The dataset included 365 white patients and 95 black women. It was observed that more white women received surgery, chemotherapy, or surgery chemotherapy sequence. It was also revealed that despite receiving the same treatment sequence, black women had higher mortality rates from ovarian cancer.

Clinicians and researchers from Indian hospitals also conducted statistical tests for survival analysis of advanced epithelial ovarian carcinoma patients. Viswanathan et al. [26] analyzed the data of stage 3 or stage 4 advanced epithelial carcinoma patients diagnosed in years 2015-2018. 111 patients were analyzed by the authors, of which the majorities were of serous histology. Most of the patients were given NACT followed by CRS. It was observed that CRS had improved overall survival and progression-free survival. Also, patients with optimal CRS after NACT had significantly lower recurrence rates and better survival than those suboptimally cytoreduced.

Tseng et al. [27] tried to identify the risk factors in women with ovarian cancer prominent in terms of cancer recurrence. Data mining techniques were used separately using leave one out cross-validation to rank the factors. Since individual data mining techniques cannot address the problem efficiently, the authors used an ensemble approach. The ensemble approach obtained better results than the pure classification techniques, with C5.0 achieving 90\% accuracy. Various authors have also used machine learning techniques to predict ovarian cancer. Lu et al. [28] used a decision tree model and feature selection measures to predict the occurrence of ovarian cancer using different blood routine tests, chemistry, and tumor markers. Several other studies also used different classification techniques to predict survival in various types of cancer [29,30]. However, most of the studies involved online datasets confined to only a specific country or area. While some of those results can be generalized to other regions, it is a well-known fact that cancer behaves differently with different environment and socioeconomic status of the patients [8].

\section{Methodology}

The proposed methodology of the study is given in Figure 1. This study's approach is divided into three major steps, including data collection, data preprocessing, and classification. The main essence of the study is involved in the dataset used for the analysis. The proposed approach follows an integrated methodology that uses data from three different profiles. However, the medical dataset suffers from many missing and irrelevant data that cannot be directly used for classification. Hence, the second step of the approach involves preprocessing of the dataset according to their data profiles. While clinical data is prepared using standard imputation techniques, we have employed sequence mining techniques to generate treatment sequences given to the patients. Similarly, attributes measuring life quality are created to capture the overall well-being of patients. After all the preprocessing, classification techniques are applied to the integrated dataset. Each step is explained in detail in the following subsections.

4.1. Data Collection. This study is based on a dataset collected from a hospital located in New Delhi, India. The case study used for the analysis is of advanced ovarian cancer. The data was collected from the hospital manually from the files digitally stored in the hospital's repository after obtaining appropriate approval from the hospital's Scientific Committee. The study got a waiver from the IRB of the hospital due to anonymity in the use of data. Due to the ethics policies of the hospital, data cannot be shared publicly. The data collected includes three kinds of attributes-clinical attributes, treatment attributes, and comorbidities data. Clinical characteristics including CA-125 levels at the time of diagnosis, presence of ascites, grade, FIGO substage, and histology were collected and recorded for each patient. CA125 levels denote a diagnostic attribute for ovarian cancer. The presence of ascites and cancer grade define the overall extent and aggressiveness of cancer cells in the body. Higher CA-125 levels, ascites presence, and grade suggest aggressive cancer. Since the collected dataset included advanced cancer patients only, the majority of the patients had stage III or stage IV cancer. Since we have used FIGO substage, stage III 


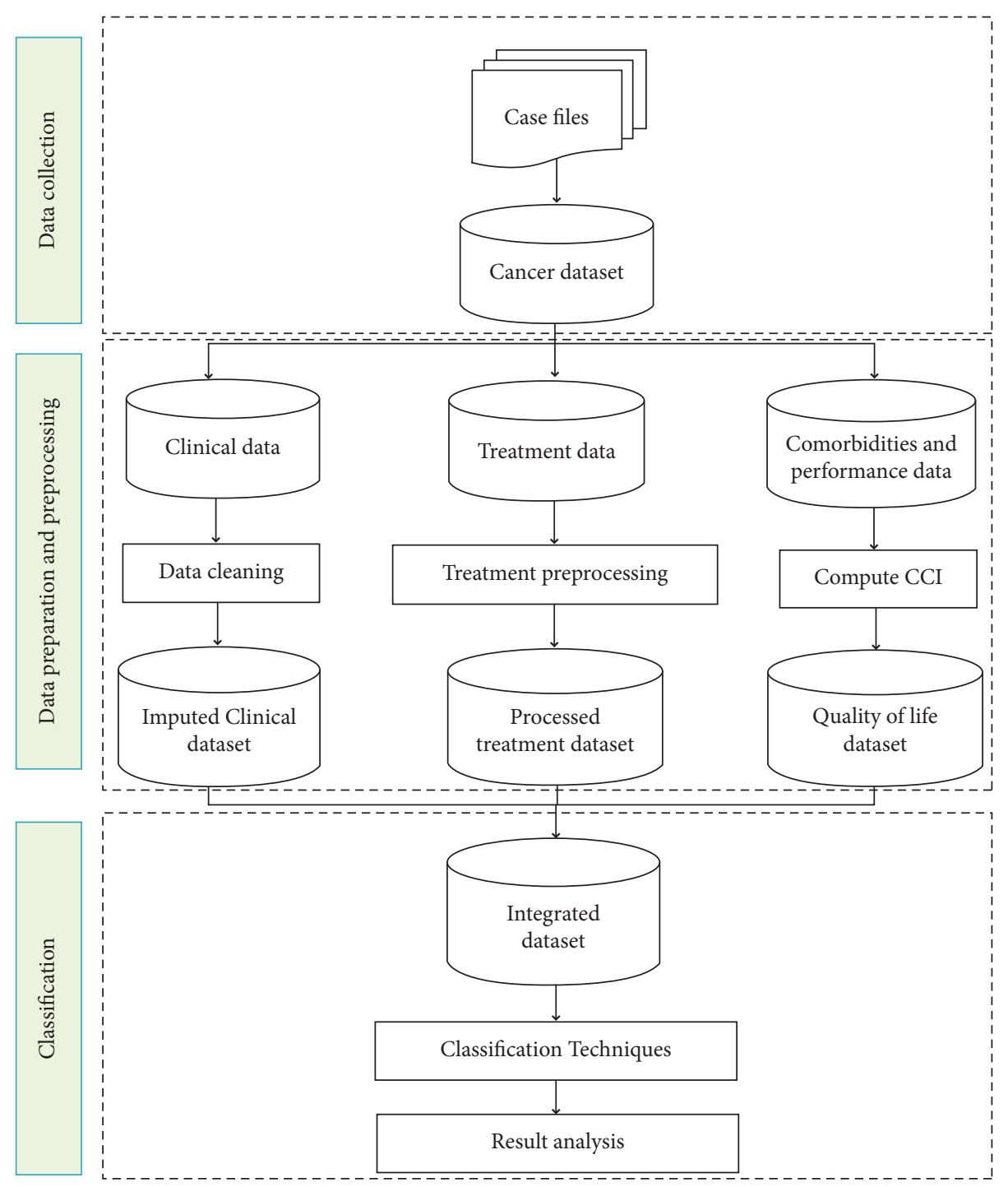

FIgURE 1: Methodology followed in study.

cancer patients were further divided into stages $3 a, 3 b$, and $3 c$ cancer. Clinical data has proven to have a high association with the survival and be the most widely used predictors in the existing studies.

Unlike the online datasets and other clinical studies, the present study also collected treatments and appropriate time intervals between each set of treatments given to each patient. The correct treatments given to the patients can prolong their survival. Also, the time elapsed between these treatments might suggest a better or worse response to the treatments. The treatments and the time intervals thus can aid a better survival model. A total of four lines of treatments were recorded for each patient. Most of the patients received less than four treatment lines for three years.

Also, ECOG levels indicating each patient's performance levels were recorded along with several comorbidities like diabetes, heart disease, and hypertension of each patient. The significance of ECOG levels and comorbidities has also been acknowledged in survival analysis of other cancer types [31]. The inclusion of life quality attributes can suggest the overall well-being of the patients and thus can better predict the overall survival of the patients. For a better comparison with existing studies and to include recent and relevant data, patients identified in the years after 2011 and before 2015 were used to collect data. The specified time range also allows for the proper retrieval of survival information of 3 years. Survival of 3 years was collected from the hospital's files or by directly contacting the patient or patient's family.

\subsection{Data Preprocessing and Analysis}

4.2.1. Data Preparation and Preprocessing. All the relevant details and information collected in the previous step were recorded and maintained in a spreadsheet. Each attribute category has been handled accordingly to gain a better perspective and improve patients' overall survival prediction.

Clinical Data Preprocessing. Clinical data has been cleaned to remove any outliers and handle missing data. Any instance with missing survival information was removed from the 
analysis to create a reliable model. Further, instances with more than fifty percent missing data were also removed as larger missing data values can lead to a weak model. The dataset after removing these patients' cases consisted of 149 patients. The rest of the missing data was handled by using mean and mode imputation techniques. Since there were only 9 cases with missing data left and mostly categorical attributes (e.g., presence of ascites), techniques like k-NN imputation did not perform well. Thus, in all the leftover instances, missing numerical attributes were filled out with the mean value of the patients' same class. Similarly, instances with missing nominal attributes were filled with the mode value of the same class. The same has been carried out with MATLAB software using rmmissing() and fillmissing() in-built methods.

Treatment Data Preprocessing. The study's objective was to process the data based on each attribute's category and behavior. Treatment preprocessing performed for this study is shown in Figure 2.

The sequences of treatments were processed by creating a database of treatment sequences for each patient. The database generated was supplied to modified sequence mining algorithm GSP. GSP was adjusted to obtain frequent treatment substrings, i.e., treatment sequences with no-gap constraint. The sequence mining algorithm has been implemented in Java [32]. 0.05 value of support has been used for the study to collect the maximum sequences of treatments possible. The no-gap constraint means that intermediate therapies would not be considered for frequent sequences; i.e., if a patient received treatment $\mathrm{W}$ in between treatments $\mathrm{X}$ and $\mathrm{Y}$, then $\mathrm{X} \longrightarrow \mathrm{Y}$ is not a valid recurring sequence. The no-gap constraint is attained at the time of counting support of each candidate sequence. The resultant treatment sequences are mentioned as follows.

The time intervals are applied in the resultant frequent treatment sequences, as shown in Figure 3. The time intervals chosen belonged to the 6 months range, i.e., $<=6$, $7-12,13-18$, till 31-36. The selection of time intervals was intuitive for 3-year survival and based on the previous literature [33]. Yet, when the data was analyzed, it was observed that most of the patients received their next treatments within 6-8 months of the previous treatment. This may be attributed to the clinical implications of treatments for advanced ovarian cancer patients to commence treatments early [13]. Thus, varying time intervals were chosen (e.g., one month, two months, and three months, till six months) to determine the prognostic value of different time intervals in ovarian cancer survival. A binary matrix is then created based on the attributes. If a patient receives a treatment $\mathrm{Y}$ within one month of treatment $\mathrm{X}$, then $\left\{\mathrm{X} \mathrm{T}_{1} \mathrm{Y}\right\}$ column will be marked 1. Since time intervals of 1 month, two months, or three months resulted in 36,18 , or 12 time ranges, it resulted in a wide array of attributes to be applied for classification. Thus, an attribute selection measure was used for the binary matrix. Information gain was computed for each attribute, and attributes having information gain greater than 0 were used for further analysis. The information gain can be calculated using the formula given by the following equations:

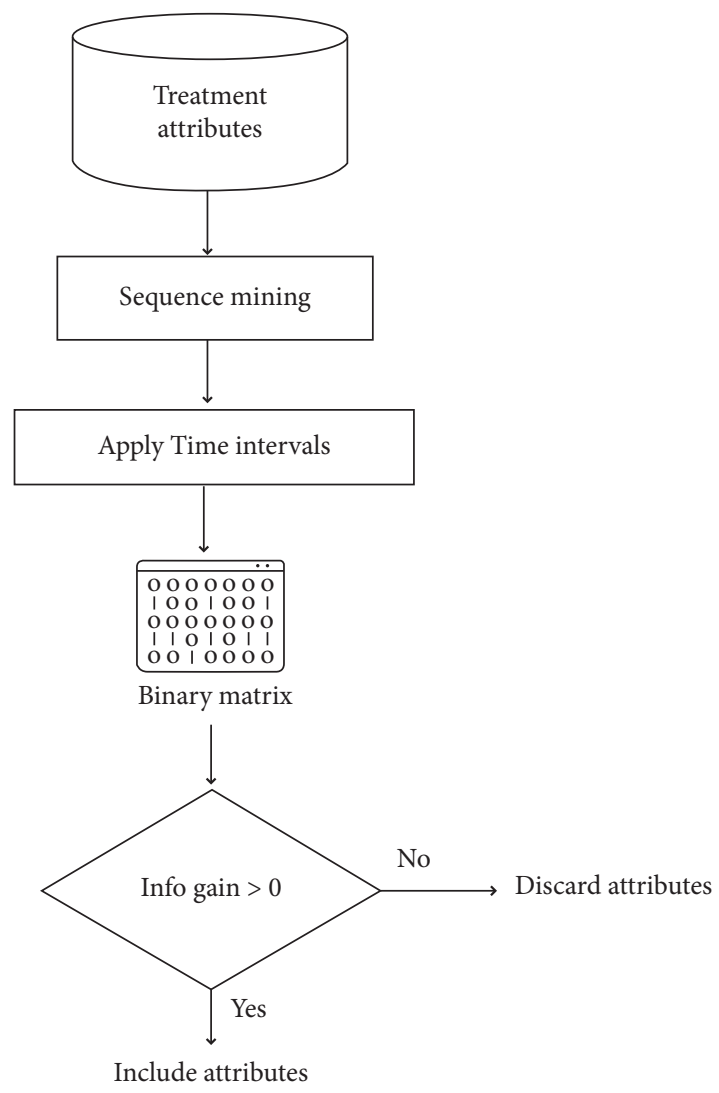

FIgURE 2: Treatment preprocessing.

$$
\begin{gathered}
\operatorname{Info}(D)=-\sum_{i=1}^{m} p_{i} \log _{2}\left(p_{i}\right), \\
\operatorname{Info}_{A}(D)=\sum_{j=1}^{v} \frac{D_{j}}{D} \times I\left(D_{j}\right), \\
\operatorname{Gain}(A)=\operatorname{Info}(D)-\operatorname{Info}_{A}(D) .
\end{gathered}
$$

Comorbidity Data Preprocessing. The comorbidities were collected for each patient as to whether she has a particular condition or not. Comorbidities like chronic obstructive pulmonary disease (COPD), diabetes, hypertension, and coronary artery disease (CAD) were recorded and correspondingly, a metric-CCI was computed for each patient. Charlson Comorbidity Index (CCI) [34] calculates a person's ten-year mortality probability by administering assigned weights to different comorbidities. The higher the computed index, the higher the probability of mortality. For instance, a person having COPD gains +1 score in his/her CCI score. Similarly, patients with uncomplicated diabetes gain an additional +1 , while an end organ damaged diabetes gains +3 score their CCI score. Thus, CCI was calculated for each patient to understand the effect of comorbidities better. A summary measure such as CCI is as good as comorbidities used to compute it. Its significance in prognosis has also been proven in the past [35]. Together with the performance 


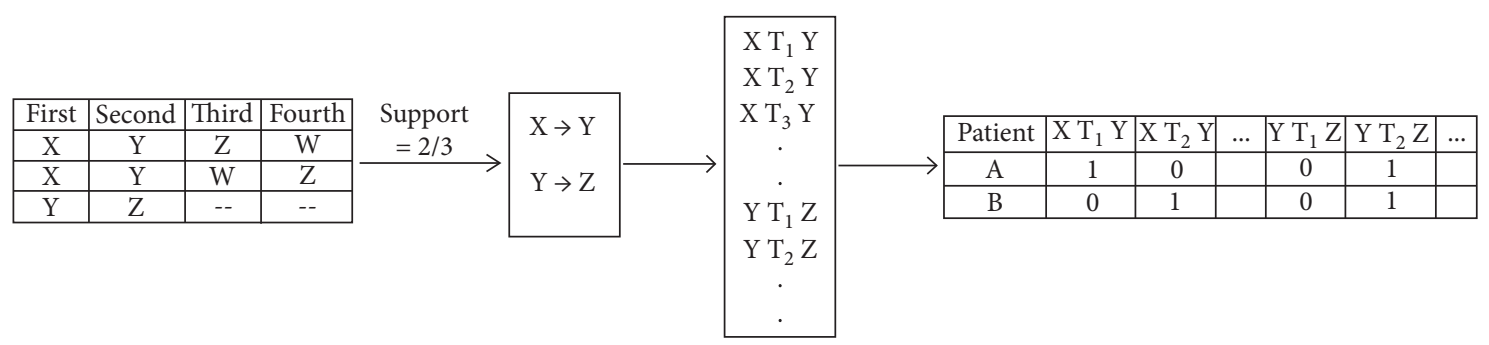

Figure 3: Time intervals in treatment sequences.

status values, CCI constitutes the quality of life part of the dataset in our study. Healthcare based IoT (IoHT) can be further utilized in collecting such life quality data in future studies [36].

4.2.2. Data Summarization and Analysis. The final set of different attributes and their description is shown in Table 1. The final dataset used for the analysis consists of 140 patients with a survival rate of $42.14 \%$ (59) and the dataset with a low degree of imbalance does not affect the predictors' performance [37]. Thus, no data balancing techniques have been employed in the study.

The present study analyzes survival based on some of the significant attributes and is shown in Figure 4.

Age has always been a controversial factor in the diagnosis and survival outcome for patients. In the present study, it is also revealed that, in younger age groups, patients have better survival outcomes than the older age group patients. However, unlike previous studies [38], ascites' presence has a somewhat opposite effect on advanced ovarian cancer patients' survival outcomes. In our dataset, patients having ascites have slightly better survival than the ones with no ascites present. Nonetheless, the existing literature did not consider the effect of ascites specifically in advanced stage. This result can be further examined by recording and assessing the ascites' volume present in future studies. CCI and ECOG, on the other hand, give promising analyses of survival outcomes. It can be seen from Figure 4 that the higher the values of CCI and ECOG, the lower the survival rate of the patients. ECOG graph shows a sharp declining trend in the chart except at ECOG performance status value 4 . This slight change in the graph is that the number of patients with ECOG status 4 was only five, and the survival rate was $0 \%$.

Similarly, a slight increase and inconsistency in the survival rate for patients with CCI score 6 are due to the small percentage of patients in that group. Thus, it is revealed from this consideration that patients with higher age, more comorbidities, and lower level of patient's general functioning are associated with lower survival rate. Other attributes like CA 125, histology, and grade did not show any relevant assessment and were not included in this study.

4.3. Classification. The integrated, processed data is supplied to classify the data into survived/deceased class. Ensemble techniques have been successfully used in various medical datasets, and thus their applicability has been tested in the present study. A statistical method, logistic regression, has also been used for comparison with the ensemble approaches.

Bagging and boosting are ensemble classifiers. Bagging or Bootstrap aggregating creates $k$ bootstrap sample datasets from the input dataset. Each test instance is classified using various base classifiers, and a combined classifier is created based on each base classifier's votes. The test instance is predicted with the class having majority votes. The averaging factor of voting helps in reducing any kind of variance in the dataset [19]. If the variance of a prediction is $\sigma^{2}$, then the variance of the average of $k$ independent predictions is reduced to $\sigma^{2} / k$. However, boosting has a weighted average effect. Boosting boosts the performance by giving more importance to instances that are difficult to classify. If a classifier incorrectly classifies an instance, the next classifier provides more significance. Thus, boosting increases that instance's weight. Boosting performs better with weak classifiers as it reduces the bias that could not be removed with bagging. Nevertheless, we may face overfitting in boosting having a weighted approach. In the present study, AdaBoost is a type of boosting algorithm and has been utilized to classify the dataset. Assuming err $\left(\mathbf{X}_{\mathbf{j}}\right)$ to be the misclassification error of tuple $X_{j}$, then the classifier $M_{i}$ error rate is the sum of the weights of the misclassified tuples as given in equation (4). The weight of a classifier $M_{i}$ 's vote will be as given in equation (5):

$$
\begin{aligned}
\operatorname{error}\left(M_{i}\right)= & \sum_{j}^{d} w_{j} \times \operatorname{err}\left(X_{j}\right), \\
& \log \frac{1-\operatorname{error}\left(M_{i}\right)}{\operatorname{error}\left(M_{i}\right)} .
\end{aligned}
$$

It has been confirmed from the previous studies that ensemble techniques, especially bagging and boosting, can perform better than most of the base classifiers individually.

When the base classifiers used for bagging are all decision tree classifiers, it is known as random forests. The forest denotes the collection of trees into a single unit (combined classifier). Random forest is called random as the decision trees are created using a random selection of attributes to decide the split at each node [39]. Each decision tree votes to determine the class of an instance, and the class with the majority votes is assigned to the test instance [40]. The random forest has proven to give better results in medical datasets. Another popular approach, called 
TABLE 1: Dataset description.

\begin{tabular}{|c|c|c|c|}
\hline & Attribute & Description & Range/values \\
\hline \multirow{13}{*}{ Clinical attributes } & Age & Age at the time of diagnosis & 17-80 (median: 54) \\
\hline & CA-125 & CA-125 value at the time of diagnosis & 8.7-16301 (median: 929.13) \\
\hline & Ascites & Presence of ascites in the bodv & Yes: 114 \\
\hline & Ascites & Presence of ascites in the body & No: 26 \\
\hline & Grade & Abnormality level of cancer cells & 2-4 (median: 3 ) \\
\hline & Stage & Figo substage & 3-4 (median: 4) \\
\hline & & & Clear cell: 1 \\
\hline & & & Endometrioid: 4 \\
\hline & & & Serous: 111 \\
\hline & Histology & & Small cell:1 \\
\hline & & Microscopic regularity of cancer cells & Germ cell: 1 \\
\hline & & & Mucinous: 6 \\
\hline & & & $\begin{array}{l}\text { Poorly/undifferentiated: } 13 \\
\text { Mixed: } 3\end{array}$ \\
\hline \multirow{7}{*}{$\begin{array}{l}\text { Treatment } \\
\text { attributes }\end{array}$} & & & Surgery $\longrightarrow$ chemotherapy \\
\hline & & & $\mathrm{NACT} \longrightarrow$ surgery \\
\hline & & & $\mathrm{NACT} \longrightarrow$ hormonal therapy \\
\hline & Treatment sequences & $\begin{array}{l}\text { Frequent treatment sequences obtained after } \\
\text { sequence mining }\end{array}$ & $\begin{array}{c}\text { Chemotherapy } \longrightarrow \text { hormonal } \\
\text { therapy }\end{array}$ \\
\hline & & & Surgery $\longrightarrow$ hormonal therapy \\
\hline & & & Chemotherapy $\longrightarrow$ CRS \\
\hline & & & Surgery $\longrightarrow$ NACT \\
\hline \multirow{2}{*}{$\begin{array}{l}\text { Life quality } \\
\text { attributes }\end{array}$} & CCI & $\begin{array}{l}\text { Charlson comorbidity index obtained using } \\
\text { comorbidities }\end{array}$ & 2-9 (median: 3 ) \\
\hline & $\begin{array}{l}\text { ECOG performance } \\
\text { status }\end{array}$ & The general well-being of a patient & 1-5 (median: 2) \\
\hline \multirow{2}{*}{ Class attribute } & \multirow{2}{*}{ Outcome } & Survival outcome after three years of cancer & Yes: 59 \\
\hline & & diagnosis & No: 81 \\
\hline
\end{tabular}
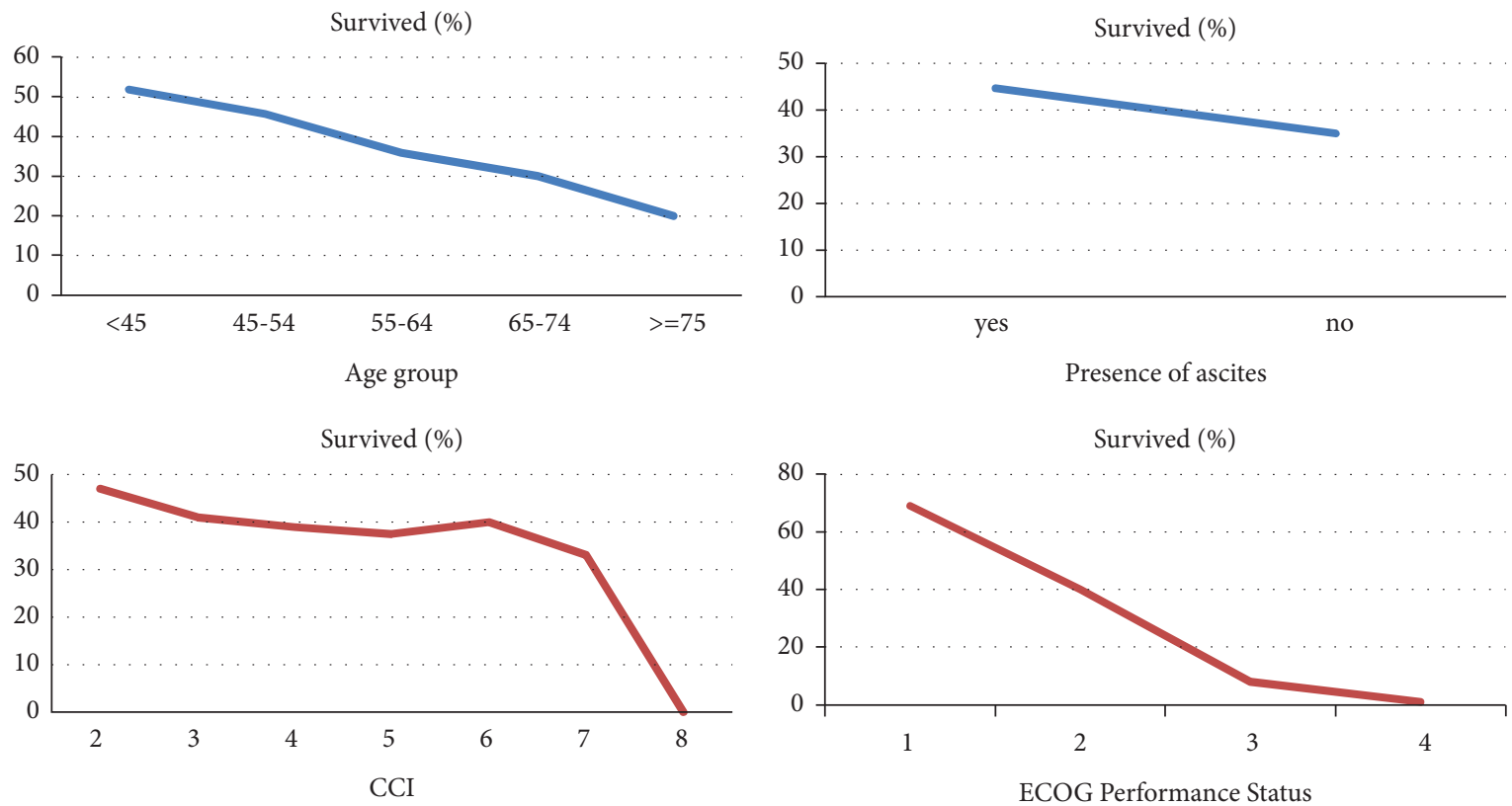

Figure 4: Data analysis with survival.

XGBoost, has been applied to the dataset using scikit-learn framework. It is a gradient tree boosting approach designed mainly to boost the speed and performance [41]. XGBoost can be used for both classification and regression problems.
It is a widely used algorithm by the researchers, specifically for scalable problems [42].

Logistic regression, being a statistical technique, has been used in the present study to compare ensemble 
techniques with statistical data mining techniques. It models the class membership probability concerning the different attributes of the dataset. It divides the dataset into two classes based on the likelihood of each instance belonging to a particular class. The probability is computed with the help of the attributes' values and estimated coefficients for each feature. The attributes are assumed to be independent to give better performance results. Logistic regression has been used by various authors in different healthcare applications to aid the diagnosis or prediction processes $[43,44]$.

When some base classifiers like decision trees, SVM, and $\mathrm{k}-\mathrm{NN}$ were used for the classification process, decision trees gave the best performance for the dataset. The performance of decision trees is acknowledged in various applications due to their high results $[45,46]$. Decision trees are also preferred and explored in many medical applications because of their simple and better clarity to the clinicians [47]. Explainable AI is yet another domain that can be explored in future studies. Thus, the classifiers used in this study utilized decision trees for creating the ensemble. Since the dataset used had a small number of instances, 10 -fold cross-validation was used for each technique. It divides the dataset into ten equal-sized partitions, where onefold acts as the testing partition, and all the other nine partitions are used for training the classifier. Thus, onefold is treated as a testing partition, and the method is fit on the remaining 9-1-fold. The results on testing partitions of 10 -fold cross-validation are averaged. Classification was performed using Classification Learner app on MATLAB software. The final experimental details with highest performance of each classifier used are as illustrated in Table 2.

\section{Result Analysis and Discussion}

Table 3 shows the results for the time interval sequence mining approach. Time ranges of two months and six months achieved the highest accuracy; thus, Table 3 shows the evaluation measures for only 2 and 6 months. Boosting achieved the best results for both the cases in terms of accuracy and AUC. ROC curves are shown in Figure 5. 5fold and 15-fold cross-validation techniques were also applied to the dataset to evaluate the methodologies. However, 10 -fold gave better results than the other two validation techniques, with the highest of $72.9 \%$ for 5 -fold and $75.4 \%$ for 15-fold. Also, as noted in the previous studies [48], ensemble techniques performed relatively better than the statistical method for our current research as well. However, it can be seen that time intervals of 2 months can better predict the survival of ovarian cancer in almost all the evaluation measures. Six-month approach gave slightly better results in specificity when only boosting is considered.

Contrary to the previous study using six months of time intervals for prostate cancer [33], we have evaluated different time intervals in our study to assess the appropriate time interval for advanced ovarian cancer patients. Thus, time intervals may vary according to cancer type as medications and cancer management differ for each cancer type. An appropriate model may have to be created according to the cancer type and possibly nature of cancer.
TABLE 2: Experimental details.

\begin{tabular}{lc}
\hline Model & Parameter settings \\
\hline Bagging & Method $=$ decision trees \\
& $\begin{array}{c}\text { Max number of splits }=139 \\
\text { Learning rate }=0.1\end{array}$ \\
\hline Boosting & $\begin{array}{c}\text { Ensemble method }=\text { AdaBoost } \\
\text { Max number of splits }=20 \\
\text { Learning rate }=0.1\end{array}$ \\
\hline Random forest & Random number seed $=0$ \\
\hline XGBoost & Maximum depth $=$ unlimited \\
Logistic regression & Maximum number of trees $=100$ \\
\hline
\end{tabular}

The treatment attributes selected for 2 months and 6 months are as given in Table 4. It is further revealed from the Table that the hypothesis in the present study that 6 months' interval might not be useful in the ovarian cancer dataset is true. The attributes selected in 6 months' intervals are only two, with both having T1 (0-6 months) intervals. Conversely, the attributes selected in 2 months' intervals are four and having varying intervals from $\mathrm{T} 1$ to $\mathrm{T} 5$ only. Thus, it might be possible that only a few, if any, patients received the next line of treatments after say 8-10 months of the previous treatments and, consecutively, did not have any significant role in survival prediction.

Further, each data profile's significance is examined by applying classification techniques to each category of attributes separately for a 2-month time interval integrated dataset. Table 5 shows the accuracy (in \%) along with the classification technique for each data profile.

It is revealed from the results that when an individual category is considered, the life quality dataset performs better than the other data profiles. Also, the treatment dataset gave better accuracy than the clinical dataset. Thus, the treatments' sequences and the time elapsed in the treatments can give the clinicians and patients better knowledge of patients' survival outcomes. This result contributes to the current understanding that, for advanced ovarian carcinoma patients, clinical attributes like CA-125, grade, etc. can indicate selecting the appropriate treatment for the patient. Still, it might not be a good indicator for survival prediction of the patient. Nonetheless, treatment sequences and mostly life quality attributes can be better used in predicting survival outcome.

5.1. Comparison of Proposed Work with Existing Literature. To determine the importance of sequence and time between different therapies given to a patient, we have further compared the proposed approach without sequence mining. A binary matrix has been created for the same, based on the medications received by each patient, irrespective of the sequence in which she received the therapy. An example of such a matrix is shown in Figure 6.

The comparison of various evaluation measures for all the approaches is shown in Table 6. Here, time interval approach attained better results than without sequence approach in all the criteria. However, specificity is the same 
TABle 3: Classification results.

\begin{tabular}{|c|c|c|c|c|c|}
\hline & & Accuracy (\%) & True positive rate or sensitivity & Specificity & Area under curve \\
\hline \multirow{5}{*}{6 months } & Bagging & 71.4 & 0.79 & 0.61 & 0.80 \\
\hline & Random forest & 70.7 & 0.64 & 0.8 & 0.72 \\
\hline & Boosting & 73.6 & 0.69 & 0.8 & 0.81 \\
\hline & Logistic regression & 65.7 & 0.68 & 0.63 & 0.70 \\
\hline & XGBoost & 71.42 & 0.71 & 0.64 & 0.78 \\
\hline \multirow{5}{*}{2 months } & Bagging & 74.3 & 0.85 & 0.59 & 0.82 \\
\hline & Random forest & 75.7 & 0.72 & 0.81 & 0.82 \\
\hline & Boosting & 76.4 & 0.80 & 0.71 & 0.85 \\
\hline & Logistic regression & 67.1 & 0.64 & 0.71 & 0.70 \\
\hline & XGBoost & 73.8 & 0.73 & 0.63 & 0.79 \\
\hline
\end{tabular}

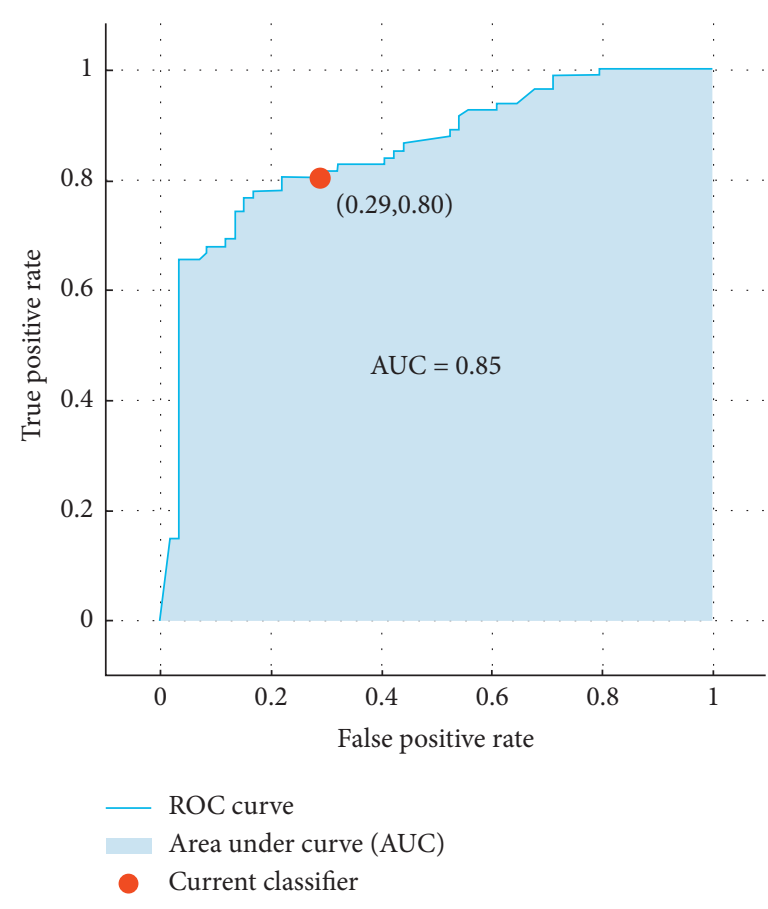

(a)

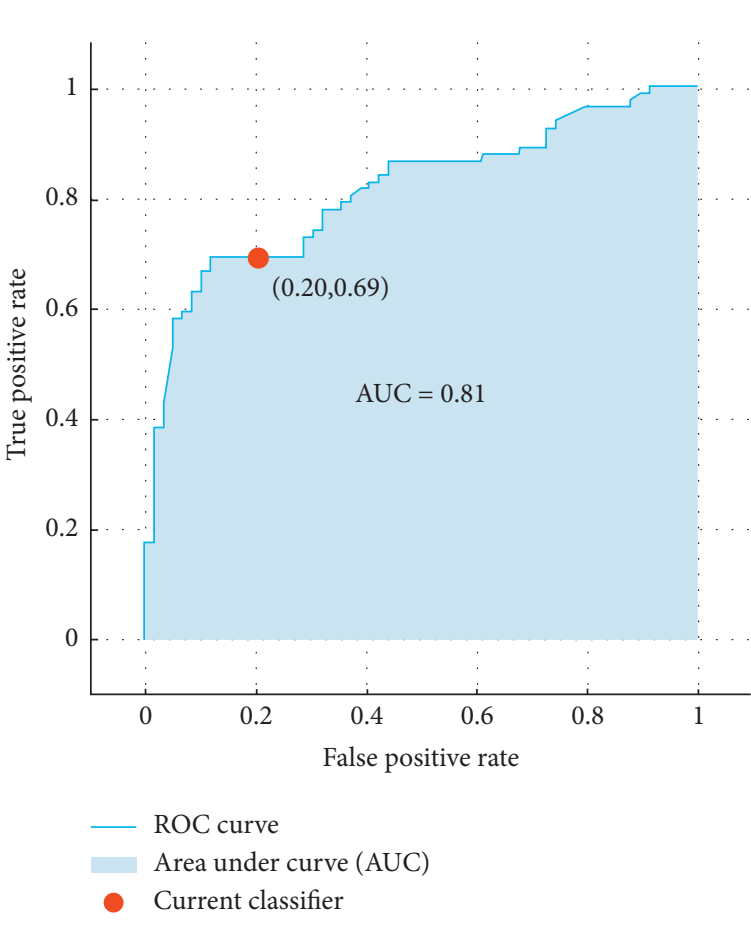

(b)

Figure 5: ROC curves for (a) boosting in 2 months' time interval; (b) boosting in 6 months' time interval.

TABLE 4: Treatment attributes selected.

\begin{tabular}{lccc}
\hline \multicolumn{2}{c}{2 months' time interval } & 6 months' time interval & Attributes \\
\hline Attributes & Information gain & Chemotherapy_T1_hormonal therapy & Information gain \\
\hline Chemotherapy_T5_CRS & 0.0458 & NACT_T1_hormonal therapy & 0.0408 \\
\hline Surgery_T5_chemotherapy & 0.0272 & & 0.008 \\
\hline Chemotherapy_T4_CRS & 0.023 & & \\
NACT_T1_hormonal therapy & 0.01 & & \\
\hline
\end{tabular}

TABLE 5: Classification results for each data profile.

\begin{tabular}{lc}
\hline Data profile & Highest accuracy in \% (classifier) \\
\hline Clinical dataset & 61.4 (bagging) \\
Treatment dataset & 65 (boosting) \\
Life quality dataset & 71.4 (boosting) \\
\hline
\end{tabular}




\begin{tabular}{|c|c|c|c|c|}
\hline Patient & First & Second & Third & Fourth \\
\hline $\mathrm{A}$ & $\mathrm{X}$ & $\mathrm{Y}$ & $\mathrm{Z}$ & -- \\
\hline $\mathrm{B}$ & $\mathrm{X}$ & $\mathrm{Y}$ & $\mathrm{W}$ & -- \\
\hline
\end{tabular}$\longrightarrow$\begin{tabular}{|c|c|c|c|c|c|}
\hline Patient & Treatment $\mathrm{X}$ & Treatment $\mathrm{Y}$ & Treatment Z & Treatment W \\
\hline $\mathrm{A}$ & 1 & 1 & 1 & 0 \\
\hline $\mathrm{B}$ & 1 & 1 & 0 & 1 \\
\hline
\end{tabular}

FIGURE 6: Without sequence treatment processing.

TABLE 6: Comparison of results.

\begin{tabular}{|c|c|c|c|c|}
\hline & Accuracy & Sensitivity or true positive rate & Specificity & Area under curve \\
\hline Without sequence mining & 0.707 & 0.78 & 0.71 & 0.77 \\
\hline 2-month time interval & 0.764 & 0.80 & 0.71 & 0.85 \\
\hline 6-month time interval & 0.736 & 0.69 & 0.8 & 0.81 \\
\hline
\end{tabular}

for two-month time interval and no-sequence mining approach. But the overall results improved in the time interval approach. The graphical representation of the results is shown in Figure 7. Also, the random forest gave better results than bagging and boosting in without sequence mining approach. Here, also, the parameter settings for random forest were the same as in the case of proposed approach (i.e., random number seed $=0$ and maximum depth $=$ unlimited). Thus, the results for only random forest have been presented in the results. The significance of time in specific treatments has also been acknowledged in previous literature on advanced epithelial ovarian cancer [13]. Hofstetter et al. [49] also demonstrated the use of intervals between surgery and chemotherapy in advanced ovarian cancer patients using statistical techniques. They also revealed that the periods were around 3-6 weeks. The present study also gave better results when time intervals of 2 months were used for the survival prediction. The results were validated statistically by computing $t$-score and corresponding $p$-values with a significance level of 0.05 . Since the best results were given by 2 -month time interval approach, it was compared with 'without sequence mining' approach. The results are given in Table 7 , and it is evident that the result is significant at $p<0.05$.

We have additionally generated an assessment of some of the recent studies to compare the data profiles and techniques used in the present study with existing literature, given in Table 8 . It can be observed from Table 8 that the majority of the studies used only clinical and treatment data for survival prediction, where treatment data mostly includes details of primary treatment only. Malhotra et al. [56] used treatment sequences along with clinical and genetic data, though the authors did not consider the time elapsed between the treatments. Also, it can be seen from Table 5 that life quality data has a significant contribution to the survival prediction, which is lacking in [56]. The collection and analyses of genetic data, however, can be the future work for the study. Studies using neural networks and deep learning are also becoming more common now with genetic and multimodal data and thus, can be utilized in future studies [62-64]. These have been further explored in various image based datasets as well for the detection and prediction purposes [65-67]. Deep learning technology has been proven in various studies to outperform basic machine learning techniques $[68,69]$. However, the dataset in the

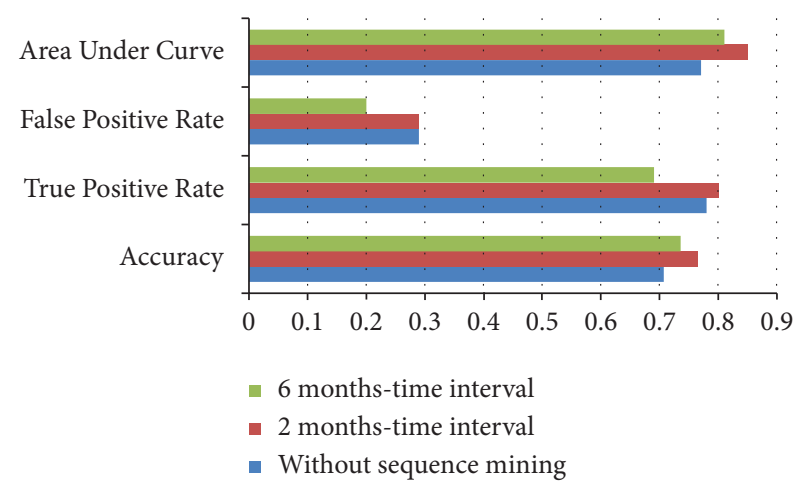

Figure 7: Comparison of results.

TABle 7: Statistical significance.

\begin{tabular}{lc}
\hline Approach & '2-months time interval' with 'without sequence mining' \\
\hline$t$-value & 1.90429 \\
$p$ value & 0.036491 \\
\hline
\end{tabular}

present study has a smaller number of instances than the existing literature, and deep learning can perform better with large amounts of training data. Due to lack of significant training data, deep learning could not be explored in this study. But this is because the present study involves recent records and only advanced-stage patients. Since earlier stages of almost all cancer already have around $90 \%$ survival rates, survival prediction is an easier task. But in the later stages, the survival rates vary from about $10 \%$ to $40 \%$. Thus, the present study creates a model established on the cancer behavior (for advanced stage only) that will be more useful for clinicians in examining the survival of cancer patients [70]. It can be observed from Table 8 that almost all the studies used dataset of all the stages. Guo et al. [57] considered earlier cancer patients for the survival prediction and achieved high results. However, as already discussed, earlier stages have considerably higher survival rates and is mostly easier to predict. Thus, more research on advanced cancer patients needs to be conducted to further compare the results.

Thus, it can be concluded from the results that the time interval approach gave better results than no-sequence approach. The time intervals may vary, but the time between 
TABLE 8: Comparison of techniques with previous literature.

\begin{tabular}{|c|c|c|c|c|c|c|c|}
\hline S.no. & Authors & Dataset & $\begin{array}{l}\text { Type of cancer } \\
\text { with stage }\end{array}$ & $\begin{array}{l}\text { Stage of } \\
\text { cancer } \\
\text { patients } \\
\text { used } \\
\end{array}$ & Type of attributes & $\begin{array}{l}\text { Classification } \\
\text { technique used }\end{array}$ & Results \\
\hline 1. & $\begin{array}{l}\text { Matsuo } \\
\text { et al. [50] }\end{array}$ & $\begin{array}{l}\text { Clinical-768 } \\
\text { patients }\end{array}$ & $\begin{array}{l}\text { Cervical } \\
\text { cancer }\end{array}$ & All stage & $\begin{array}{l}\text { (i) Clinical } \\
\text { (ii) Treatment }\end{array}$ & $\begin{array}{c}\text { Deep learning and } \\
\text { cox proportional } \\
\text { model }\end{array}$ & $\begin{array}{c}\text { Mean absolute error of } \\
30.7 \text { (deep learning), } \\
43.6 \text { (cox proportional } \\
\text { hazard regression) }\end{array}$ \\
\hline 2. & $\begin{array}{c}\text { Park et al. } \\
{[51]}\end{array}$ & SEER dataset & Breast cancer & All stage & $\begin{array}{l}\text { (i) Clinical } \\
\text { (ii) Treatment }\end{array}$ & Subgroup mining & $\begin{array}{c}\text { Effective rules } \\
\text { generated }\end{array}$ \\
\hline 3. & $\begin{array}{l}\text { Simsek } \\
\text { et al. [29] }\end{array}$ & SEER dataset & Breast cancer & All stage & (i) Clinical & $\begin{array}{l}\text { ANNs and logistic } \\
\text { regression }\end{array}$ & $\begin{array}{c}\text { 83.6\% (ANNs) } \\
82.9 \%(\mathrm{LR}) \\
\text { for 5-year survival } \\
\end{array}$ \\
\hline 4. & $\begin{array}{l}\text { Wang et al. } \\
\text { [52] }\end{array}$ & $\begin{array}{l}\text { Clinical-1075 } \\
\text { patients }\end{array}$ & Lung cancer & All stage & $\begin{array}{l}\text { (i) Clinical } \\
\text { (ii) Treatment } \\
\text { (iii) Comorbidities }\end{array}$ & $\begin{array}{c}\text { Gaussian bayesian } \\
\text { network }\end{array}$ & $\begin{array}{c}R^{2} \text { of } 93.57 \% \text { (stage-I), } \\
86.83 \% \text { (stage-II), } \\
67.22 \% \text { (stage-III), } \\
52.94 \% \text { (stage-IV) }\end{array}$ \\
\hline 5. & $\begin{array}{l}\text { García- } \\
\text { Laencina } \\
\text { et al. [53] }\end{array}$ & $\begin{array}{l}\text { Clinical-399 } \\
\text { patients }\end{array}$ & Breast cancer & All stage & $\begin{array}{l}\text { (i) Clinical } \\
\text { (ii) Treatment }\end{array}$ & $\begin{array}{l}\text { KNN, logistic } \\
\text { regression, } \\
\text { decision trees, } \\
\text { support vector } \\
\text { machine }\end{array}$ & $81 \%$ (highest in $\mathrm{KNN}$ ) \\
\hline 6. & $\begin{array}{l}\text { Toth et al. } \\
\quad[54]\end{array}$ & $\begin{array}{c}\text { National } \\
\text { health } \\
\text { database- } \\
28817 \text { patients }\end{array}$ & Colon cancer & All stage & (i) Treatment & Sequence mining & - \\
\hline 7. & $\begin{array}{c}\text { Koo et al. } \\
{[30]}\end{array}$ & $\begin{array}{c}\text { Clinical-7267 } \\
\text { patients }\end{array}$ & $\begin{array}{c}\text { Prostate } \\
\text { cancer }\end{array}$ & All stage & $\begin{array}{l}\text { (i) Clinical } \\
\text { (ii) Treatment }\end{array}$ & $\begin{array}{l}\text { Artificial neural } \\
\text { networks }\end{array}$ & $\begin{array}{c}84.9 \% \text { overall } 5 \text {-year } \\
\text { survival }\end{array}$ \\
\hline 8. & $\begin{array}{l}\text { Kate and } \\
\text { Nadig [55] }\end{array}$ & SEER dataset & Breast cancer & All stage & $\begin{array}{l}\text { (i) Clinical } \\
\text { (ii) Treatment }\end{array}$ & $\begin{array}{l}\text { Logistic } \\
\text { regression, naïve } \\
\text { bayes, decision } \\
\text { tree }\end{array}$ & $84.2 \%$ (naïve bayes) \\
\hline 9. & $\begin{array}{l}\text { Malhotra } \\
\text { et al. [56] }\end{array}$ & $\begin{array}{l}\text { Clinical-393 } \\
\text { patients }\end{array}$ & $\begin{array}{l}\text { Glioblastoma } \\
\text { cancer }\end{array}$ & All stage & $\begin{array}{l}\text { (i) Treatment } \\
\text { (ii) Genetic } \\
\text { (iii) Clinical }\end{array}$ & $\begin{array}{c}\text { Sequence mining } \\
\text { with statistical } \\
\text { techniques }\end{array}$ & $\begin{array}{l}85 \% \text { (logistic } \\
\text { regression) }\end{array}$ \\
\hline 10. & $\begin{array}{l}\text { Guo et al. } \\
\text { [57] }\end{array}$ & $\begin{array}{l}\text { Clinical-5842 } \\
\text { patients }\end{array}$ & $\begin{array}{l}\text { Cervical } \\
\text { cancer }\end{array}$ & $\begin{array}{l}\text { Stage IA1 to } \\
\text { IIB2 }\end{array}$ & (i) Clinical & $\begin{array}{l}\text { SVM, decision } \\
\text { tree, random } \\
\text { forest, } A N N \text { etc. }\end{array}$ & $\begin{array}{l}0.895 \text { and } 0.89 \text { AUC } \\
\text { (light GBM and } \\
\text { random forest) }\end{array}$ \\
\hline 11. & $\begin{array}{l}\text { Kalafi et al. } \\
\quad[58]\end{array}$ & $\begin{array}{l}\text { University } \\
\text { Malaya } \\
\text { medical } \\
\text { cancer } \\
\text { registry-8066 } \\
\text { patients } \\
\end{array}$ & Breast cancer & All stage & (ii) Treatment & $\begin{array}{l}\text { SVM, MLP } \\
\text { (multilayer } \\
\text { perceptron), } \\
\text { decision trees, } \\
\text { random forest }\end{array}$ & $88.2 \%$ accuracy (MLP) \\
\hline 12. & $\begin{array}{l}\text { Alabi et al. } \\
\quad[59]\end{array}$ & SEER dataset & Oral cancer & All stage & (i) Clinical & $\begin{array}{l}\text { Logistic } \\
\text { regression, SVM, } \\
\text { bayes point, } \\
\text { boosting, decision } \\
\text { forest, decision } \\
\text { jungle }\end{array}$ & 88.7\% (boosting) \\
\hline 13. & $\begin{array}{l}\text { Bos et al. } \\
{[60]}\end{array}$ & $\begin{array}{l}\text { Clinical-177 } \\
\text { patients }\end{array}$ & Oral cancer & All stage & $\begin{array}{c}\text { (i) Clinical } \\
\text { (ii) Radiomic (MRI) }\end{array}$ & Logistic regression & 0.744 AUC \\
\hline 14. & $\begin{array}{l}\text { Hira et al. } \\
{[61]}\end{array}$ & $\begin{array}{l}\text { TCGA-579 } \\
\text { and } 593 \\
\text { samples }\end{array}$ & $\begin{array}{l}\text { Ovarian } \\
\text { cancer }\end{array}$ & All stage & (i) Multi-omics data & Deep learning & $\begin{array}{c}93.2-95.5 \% \text { and } \\
87.1-95.7 \% \text { accuracy }\end{array}$ \\
\hline 15. & $\begin{array}{l}\text { Proposed } \\
\text { approach }\end{array}$ & $\begin{array}{l}\text { Clinical-140 } \\
\text { patients }\end{array}$ & $\begin{array}{l}\text { Ovarian } \\
\text { cancer }\end{array}$ & $\begin{array}{l}\text { Advanced } \\
\text { stage }\end{array}$ & $\begin{array}{c}\text { (i) Clinical } \\
\text { (ii) Treatment } \\
\text { (iii) Life quality } \\
\text { (comorbidities + ECOG) }\end{array}$ & $\begin{array}{l}\text { Sequence mining } \\
\text { with ensemble }\end{array}$ & $\begin{array}{l}\text { 76.4\% accuracy and } \\
0.85 \text { AUC (boosting) }\end{array}$ \\
\hline
\end{tabular}


treatments can also create a better and reliable predictive model for other cancer patients. The integrated dataset, including data from all profiles, is a better prediction model than the existing models, including only clinical attributes and treatment attributes with no frequent sequences. The clinicians can use this information while deciding the appropriate treatments for advanced ovarian carcinoma patients and the elapsed time between each treatment. The patients' general well-being can also be useful indicators in determining the treatments and corresponding overall survivability of the patients.

\section{Conclusion}

Advanced ovarian carcinoma patients have a poor prognosis compared to early-stage patients. The present study gives some worthwhile comprehensions in advanced ovarian cancer survival. An integrated predictive model has been created using three different data profiles from a realworld clinical dataset. It also focuses on the significance of treatment sequences with varying time elapsed between treatments and various life quality attributes in the survival analysis of patients. Cancer patients are often treated with multiple lines of therapy. The present study validates and ascertains the use of varying time elapsed between treatments in examining the survival of patients using a modified sequential mining algorithm of GSP, and various machine learning techniques. It was revealed that life quality attributes and treatment sequences with the time intervals could predict survival better than clinical facts. Also, time intervals of two months between the treatment sequences performed better than other time intervals with an accuracy of $76.4 \%$ and 0.85 AUC. The proposed approach of modified sequential mining algorithm and classification with $76.4 \%$ accuracy performed better than the existing approach without sequential mining, giving around $70 \%$ accuracy. The results were also statistically validated. Thus, the clinicians and researchers should consider patients' quality of life and line of treatments with time elapsed between them while creating a predictive model for cancer patients.

However, there are a few limitations and possible future aspects worth noting. This study used data from only five years of the hospital to record current medications and other medical technologies. The dataset thus had a small number of instances, which could have resulted in overfitting in classification. Also, the dataset was collected manually by the authors. Some recording errors might have been created in the data. Besides, precise medications and chemotherapy cycles or dosage were not considered to avoid confusion. Yet, these might be useful if we had a larger dataset.

\section{Data Availability}

The study was made possible with the dataset collected from Rajiv Gandhi Cancer Institute \& Research Center, New Delhi.

\section{Conflicts of Interest}

The authors declare that they have no conflicts of interest.

\section{Acknowledgments}

The authors acknowledge and extend their appreciation to the Researchers Supporting Project number (RSP-2021/124), King Saud University, Riyadh, Saudi Arabia, for funding this study.

\section{References}

[1] The Top 10 Causes of Death, WHO, Geneva, Switzerland, Available at: https://www.who.int/news-room/fact-sheets/ detail/the-top-10-causes-of-death, 2020.

[2] R. J. Kate and R. Nadig, "Stage-specific predictive models for breast cancer survivability," International Journal of Medical Informatics, vol. 97, pp. 304-311, 2017.

[3] S. Walczak and V. Velanovich, "Improving prognosis and reducing decision regret for pancreatic cancer treatment using artificial neural networks," Decision Support Systems, vol. 106, pp. 110-118, 2018.

[4] K. Juneja and C. Rana, "An improved weighted decision tree approach for breast cancer prediction," International Journal of Information Technology, vol. 12, no. 3, pp. 797-804, 2020.

[5] H. M. Zolbanin, D. Delen, and A. Hassan Zadeh, "Predicting overall survivability in comorbidity of cancers: a data mining approach," Decision Support Systems, vol. 74, pp. 150-161, 2015.

[6] "Surveillance, epidemiology, and end results (SEER) Program," 1975, https://www.seer.cancer.gov.

[7] T. R. Network, The Cancer Genome Atlas Data Portal, National Institute of Health, Maryland, USA, 2010.

[8] M. Z. Nezhad, N. Sadati, K. Yang, and D. Zhu, "A Deep Active Survival Analysis approach for precision treatment recommendations: application of prostate cancer," Expert Systems with Applications, vol. 115, pp. 16-26, 2019.

[9] C. A. Hamilton, A. Miller, Y. Casablanca et al., "Clinicopathologic characteristics associated with long-term survival in advanced epithelial ovarian cancer: an NRG Oncology/ Gynecologic Oncology group ancillary data study," Gynecologic Oncology, vol. 148, no. 2, pp. 275-280, 2018.

[10] L. Vincent, C. Jankowski, L. Ouldamer et al., "Prognostic factors of overall survival for patients with FIGO stage IIIc or IVa ovarian cancer treated with neo-adjuvant chemotherapy followed by interval debulking surgery: a multicenter cohort analysis from the FRANCOGYN study group," European Journal of Surgical Oncology, vol. 46, no. 9, pp. 1689-1696, 2020.

[11] J. S. Dungan, "Neoadjuvant chemotherapy or primary surgery in stage IIIC or IV ovarian cancer," Year Book of Obstetrics, Gynecology, and Women's Health, vol. 2011, pp. 507-508, 2011.

[12] S. Akhavan, A. Ghahghaei-Nezamabadi, M. Modaresgilani et al., "Impact of diabetes mellitus on epithelial ovarian cancer survival," BMC Cancer, vol. 18, no. 1, 2018.

[13] A. Maheshwari, N. Kumar, S. Gupta et al., "Outcomes of advanced epithelial ovarian cancer treated with neoadjuvant chemotherapy," Indian Journal of Cancer, vol. 55, no. 1, pp. 50-54, 2018.

[14] Y. Zhang, G. Luo, M. Li et al., "Global patterns and trends in ovarian cancer incidence: age, period and birth cohort analysis," BMC Cancer, vol. 19, no. 1, p. 984, 2019. 
[15] S. B. Coburn, F. Bray, M. E. Sherman, and B. Trabert, "International patterns and trends in ovarian cancer incidence, overall and by histologic subtype," International Journal of Cancer, vol. 140, no. 11, pp. 2451-2460, 2017.

[16] R. Takiar, "Status of ovarian cancer in India (2012-14)," EC Gynaecology, vol. 8, no. 5, pp. 358-364, 2019.

[17] N. Bhatla, "The world ovarian cancer coalition atlas: global trends in incidence, mortality and survival," 2018.

[18] S. Sato and H. Itamochi, "Neoadjuvant chemotherapy in advanced ovarian cancer: latest results and place in therapy," Therapeutic advances in medical oncology, vol. 6, no. 6, pp. 293-304, 2014.

[19] M. J. Zaki and W. Meira, Data Mining and Analysis, Cambridge University Press, Cambridge, UK, 2014.

[20] R. Srikant and R. Agrawal, "Mining sequential patterns: generalizations and performance improvements," in Proceedings of the 5th International Conference on Extending Database Technology: Advances in Database Technology (EDBT '96), P. M. G. Apers, M. Bouzeghoub, and G. Gardarin, Eds., pp. 3-17, Springer-Verlag, London, UK, 1996.

[21] R. Agrawal and R. Srikant, "Fast algorithms for mining association rules," in Proceedings of the 20th International Conference on Very Large Data Bases, VLDB, Santiago, Chile, September 1994.

[22] M. J. Zaki, "SPADE: an efficient algorithm for mining frequent sequences," Machine Learning, vol. 42, no. 1/2, pp. 31-60, 2001.

[23] P. Jian Pei, H. Jiawei Han, B. Mortazavi-Asl et al., "PrefixSpan: mining sequential patterns efficiently by prefix-projected pattern growth," in Proceedings of the 17th International Conference on Data Engineering, Heidelberg, Germany, April 2001.

[24] K. Deng, C. Yang, Q. Tan et al., "Sites of distant metastases and overall survival in ovarian cancer: a study of 1481 patients," Gynecologic Oncology, vol. 150, no. 3, pp. 460-465, 2018.

[25] J. S. Hildebrand, K. Wallace, W. S. Graybill, and L. E. Kelemen, "Racial disparities in treatment and survival from ovarian cancer," Cancer Epidemiology, vol. 58, pp. 77-82, 2019.

[26] M. P. Viswanathan, D. S. Kumar, P. Muniasamy, S. Navin Noushad, and D. Pradeep, "Patterns of relapse and survival analysis of advanced epithelial ovarian cancers operated in a tertiary cancer centre," Indian Journal of Gynecologic Oncology, vol. 17, p. 72, 2019.

[27] C.-J. Tseng, C.-J. Lu, C.-C. Chang, G.-D. Chen, and C. Cheewakriangkrai, "Integration of data mining classification techniques and ensemble learning to identify risk factors and diagnose ovarian cancer recurrence," Artificial Intelligence in Medicine, vol. 78, pp. 47-54, 2017.

[28] M. Lu, Z. Fan, B. Xu et al., "Using machine learning to predict ovarian cancer," International Journal of Medical Informatics, vol. 141, Article ID 104195, 2020.

[29] S. Simsek, U. Kursuncu, E. Kibis, M. AnisAbdellatif, and A. Dag, "A hybrid data mining approach for identifying the temporal effects of variables associated with breast cancer survival," Expert Systems with Applications, vol. 139, Article ID 112863, 2019.

[30] K. C. Koo, K. S. Lee, S. Kim et al., "Long short-term memory artificial neural network model for prediction of prostate cancer survival outcomes according to initial treatment strategy: development of an online decision-making support system," World Journal of Urology, vol. 38, no. 10, pp. 2469-2476, 2020.
[31] M. N. Doja, I. Kaur, and T. Ahmad, "Age-specific survival in prostate cancer using machine learning," Data Technologies and Applications, vol. 54, no. 2, pp. 215-234, $2020 \mathrm{~b}$.

[32] P. Fournier-Viger, J. C. W. Lin, A. Gomariz et al., "The SPMF open-source data mining library version 2," in Proceedings of the Joint European Conference on Machine Learning and Knowledge Discovery in Databases, September 2016.

[33] I. Kaur, M. N. Doja, and T. Ahmad, "Time-range based sequential mining for survival prediction in prostate cancer," Journal of Biomedical Informatics, vol. 110, Article ID 103550, 2020.

[34] S. Schneeweiss, P. S. Wang, J. Avorn, and R. J. Glynn, "Improved comorbidity adjustment for predicting mortality in Medicare populations," Health Services Research, vol. 38, no. 4, pp. 1103-1120, 2003.

[35] S. R. Austin, Y. N. Wong, R. G. Uzzo, J. R. Beck, and B. L. Egleston, "Why summary comorbidity measures such as the Charlson comorbidity index and elixhauser score work," Medical Care, vol. 53, no. 9, pp. e65-e72, 2015.

[36] E. M. Abou-Nassar, A. M. Iliyasu, P. M. El-Kafrawy, O.-Y. Song, A. K. Bashir, and A. A. A. El-Latif, "DITrust chain: towards blockchain-based trust models for sustainable healthcare IoT systems," IEEE Access, vol. 8, 2020.

[37] M. Hammad, M. H. Alkinani, B. B. Gupta, and A. A. Abd ElLatif, "Myocardial infarction detection based on deep neural network on imbalanced data," Multimedia Systems, 2021.

[38] E. Kipps, D. S. Tan, and S. B. Kaye, "Meeting the challenge of ascites in ovarian cancer: new avenues for therapy and research," Nature Reviews Cancer, vol. 13, no. 4, pp. 273-8210, 2013.

[39] C. C. Aggarwal, Data Mining: The Textbook, Springer International Publishing, New-York, NY, USA, 2015.

[40] J. Han and M. Kamber, Data Mining Concepts and Techniques, 3rd edition, 2012.

[41] T. Chen and C. Guestrin, "XGBoost," in Proceedings of the 22nd ACM SIGKDD International Conference on Knowledge Discovery and Data Mining-KDD '16, 2016.

[42] M. Akcay, D. Etiz, and O. Celik, "Prediction of survival and recurrence patterns by machine learning in gastric cancer cases undergoing radiation therapy and chemotherapy," Advances in radiation oncology, vol. 5, no. 6, pp. 1179-1187, 2020.

[43] T. Mazzocco and A. Hussain, "Novel logistic regression models to aid the diagnosis of dementia," Expert Systems with Applications, vol. 39, no. 3, pp. 3356-3361, 2012.

[44] C. Ieracitano, N. Mammone, A. Bramanti, S. Marino, A. Hussain, and F. C. Morabito, "A Time-frequency based machine learning system for brain states classification via EEG signal processing," in Proceedings of the 2019 International Joint Conference on Neural Networks (IJCNN), Budapest, Hungary, July 2019.

[45] S. Höppner, E. Stripling, B. Baesens, S. vanden Broucke, and T. Verdonck, "Profit driven decision trees for churn prediction," European Journal of Operational Research, vol. 284, pp. 920-933, 2020.

[46] I. Kaur, G. S. Narula, and V. Jain, "Differential analysis of token metric and object oriented metrics for fault prediction," International Journal of Information Technology, vol. 9, pp. 93-100, 2017.

[47] K. Mathan, P. M. Kumar, P. Panchatcharam, G. Manogaran, and R. Varadharajan, "A novel Gini index decision tree data mining method with neural network classifiers for prediction of heart disease," Design Automation for Embedded Systems, vol. 22, pp. 225-242, 2018. 
[48] W. Książek, M. Hammad, P. Pławiak, U. R. Acharya, and R. Tadeusiewicz, "Development of novel ensemble model using stacking learning and evolutionary computation techniques for automated hepatocellular carcinoma detection," Biocybernetics and Biomedical Engineering, vol. 40, 2020.

[49] G. Hofstetter, N. Concin, I. Braicu et al., "The time interval from surgery to start of chemotherapy significantly impacts prognosis in patients with advanced serous ovarian carcinoma-analysis of patient data in the prospective OVCAD study," Gynecologic Oncology, vol. 131, no. 1, pp. 15-20, 2013.

[50] K. Matsuo, S. Purushotham, B. Jiang et al., "Survival outcome prediction in cervical cancer: cox models vs deep-learning model," American Journal of Obstetrics and Gynecology, vol. 220, no. 381, pp. e1-e14, 2019.

[51] J. V. Park, S. J. Park, and J. S. Yoo, "Finding characteristics of exceptional breast cancer subpopulations using subgroup mining and statistical test," Expert Systems with Applications, vol. 118, pp. 553-562, 2019.

[52] K. J. Wang, J. L. Chen, K. H. Chen, and K. M. Wang, "Survivability prognosis for lung cancer patients at different severity stages by a risk factor-based bayesian network modeling," Journal of Medical Systems, vol. 44, no. 3, p. 65, 2020.

[53] P. J. García-Laencina, P. H. Abreu, M. H. Abreu, and N. Afonoso, "Missing data imputation on the 5-year survival prediction of breast cancer patients with unknown discrete values," Computers in Biology and Medicine, vol. 59, pp. 125-133, 2015.

[54] K. Tóth, I. Kósa, and Á. Vathy-Fogarassy, "Frequent treatment sequence mining from medical databases," Studies in Health Technology and Informatics, vol. 236, pp. 211-218, 2017.

[55] R. J. Kate and R. Nadig, "Stage-specific predictive models for breast cancer survivability," International Journal of Medical Informatics, vol. 97, pp. 304-311, 2018.

[56] K. Malhotra, S. B. Navathe, D. H. Chau, C. Hadjipanayis, and J. Sun, "Constraint based temporal event sequence mining for Glioblastoma survival prediction," Journal of Biomedical Informatics, vol. 61, pp. 267-275, 2016.

[57] C. Guo, J. Wang, Y. Wang et al., "Novel artificial intelligence machine learning approaches to precisely predict survival and site-specific recurrence in cervical cancer: a multi-institutional study," Translational Oncology, vol. 14, no. 5, Article ID 101032, 2021.

[58] E. Y. Kalafi, N. A. M. Nor, N. A. Taib, M. D. Ganggayah, C. Town, and S. K. Dhillon, "Machine learning and deep learning approaches in breast cancer survival prediction using clinical data," Folia Biologica, vol. 65, no. 5-6, pp. 212-220, 2019.

[59] R. O. Alabi, A. A. Mäkitie, M. Pirinen, M. Elmusrati, I. Leivo, and A. Almangush, "Comparison of nomogram with machine learning techniques for prediction of overall survival in patients with tongue cancer," International Journal of Medical Informatics, vol. 145, Article ID 104313, 2021.

[60] P. Bos, M. W. M. Van Den Brekel, Z. A. R. Gouw et al., "Improved outcome prediction of oropharyngeal cancer by combining clinical and MRI features in machine learning models," European Journal of Radiology, vol. 139, Article ID 109701, 2021.

[61] M. T. Hira, M. A. Razzaque, C. Angione, G. Scrivens, S. Sawan, and M. Sarker, "Integrated multi-omics analysis of ovarian cancer using variational autoencoders," Scientific Reports, vol. 11, p. 6265, 2021.
[62] C. Ieracitano, N. Mammone, A. Hussain, and F. C. Morabito, "A novel multi-modal machine learning based approach for automatic classification of EEG recordings in dementia," Neural Networks, vol. 123, pp. 176-190, 2020.

[63] M. Mahmud, M. S. Kaiser, A. Hussain, and S. Vassanelli, "Applications of deep learning and reinforcement learning to biological data," IEEE Transactions on Neural Networks and Learning Systems, vol. 29, no. 6, pp. 2063-2079, 2018.

[64] S. Poria, E. Cambria, A. Hussain, and G.-B. Huang, "Towards an intelligent framework for multimodal affective data analysis," Neural Networks, vol. 63, pp. 104-116, 2015.

[65] A. Sedik, A. M. Iliyasu, B. Abd El-Rahiem et al., "Deploying machine and deep learning models for efficient data-augmented detection of COVID-19 infections," Viruses, vol. 12, no. 7, p. 769, 2020.

[66] A. Sedik, M. Hammad, F. E. Abd El-Samie, B. B. Gupta, and A. A. Abd El-Latif, "Efficient deep learning approach for augmented detection of coronavirus disease," Neural Computing and Applications, 2021.

[67] A. Alghamdi, M. Hammad, H. Ugail et al., "Detection of myocardial infarction based on novel deep transfer learning methods for urban healthcare in smart cities," Multimedia Tools and Applications, 2020.

[68] A. Voulodimos, N. Doulamis, A. Doulamis, and E. Protopapadakis, "Deep learning for computer vision: a brief review," Computational Intelligence and Neuroscience, vol. 2018, Article ID 7068349, 13 pages, 2018.

[69] Y. Guo, Y. Liu, A. Oerlemans, S. Lao, S. Wu, and M. S. Lew, "Deep learning for visual understanding: a review," Neurocomputing, vol. 187, pp. 27-48, 2016.

[70] M. N. Doja, I. Kaur, and T. Ahmad, "Current state of the art for survival prediction in cancer using data mining techniques," Current Bioinformatics, vol. 15, p. 174, 2020 a. 